14

\title{
Применение анализа совместных рекуррентностей к оценке фазовой синхронизации физиологических сигналов
}

\author{
(C) O.Е. Дик, ${ }^{1}$ А.Л. Глазов ${ }^{2}$ \\ ${ }^{1}$ Институт ффизиологии им. И.П. Павлова РАН, \\ 199034 Санкт-Петербург, Россия \\ ${ }^{2}$ Физико-технический институт им. А.Ф. Иофффе РАН, \\ 194021 Санкт-Петербург, Россия \\ e-mail: dickviola@gmail.com, glazov.holo@mail.ioffe.ru
}

Поступило в Редакцию 18 февраля 2021 г.

В окончательной редакции 20 июля 2021 г.

Принято к публикации 22 июля 2021 г.

На основе анализа совместных рекуррентностей выявлены различия в фазовой синхронизации между ритмической фотостимуляцией и ответами мозга у лиц с мерцательной аритмией пароксизмального и постоянного типа. В качестве меры фазовой синхронизации между двумя сигналами рассмотрен коэффициент взаимной корреляции между вероятностями рекуррентностей соответствующих фазовых траекторий. При удлинении времени существования мерцательной аритмии и возрастании степени снижения когнитивных функций значение этого коэффициента возрастает для ответов мозга на частоты тета-диапазона.

Ключевые слова: рекуррентность, ритмическая фотостимуляция, мерцательная аритмия.

DOI: $10.21883 /$ JTF.2021.12.51771.41-21

\section{Введение}

Для исследования фазовой синхронизации физиологических ритмов существует множество методов, связанных с преобразованием Гильберта [1,2], с вейвлетным преобразованием $[3,4]$, а также с анализом совместных рекуррентностей [5].

Метод анализа совместных рекуррентностей позволяет визуализировать одновременное возникновение рекуррентностей в двух временных последовательностях, полученных от различных физических источников, и определять количественные характеристики этих рекуррентностей [5]. При вычислении вероятностей возврата фазовых траекторий двух сигналов в окрестность предыдущего состояния после некоторого временного интервала возможно получение совпадения максимумов этих вероятностей. В этом случае говорят о фазовой синхронизации между двумя сигналами, в частности, сигналом возбуждения и откликом на него [6]. При анализе физиологических сигналов этот метод был применен для изучения фазовой синхронизации в паттернах биоэлектрической активности мозга, регистрируемой с поверхности головы в виде электроэнцефалограммы (ЭЭГ), у пациентов с генерализованной и фокальной формами эпилепсии [7]. Авторы работы [7] показали, что с помощью этого метода в случае глобальной эпилепсии можно различать состояния перед приступом эпилепсии и во время приступа или идентифицировать очаг эпилепсии в случае фокальной эпилепсии.

В настоящей работе этот метод будет применен для исследования синхронизации между паттернами ЭЭГ и ритмическими стимулами, т.е. при регистрации ЭЭГ в условиях функциональной нагрузки в виде ритмической фотостимуляции у пациентов с нарушениями сердечного ритма в виде мерцательной аритмии (пароксизмальной или постоянной формах фибрилляции предсердий).

В ряде клинических работ установлены взаимосвязи между аритмией и снижением когнитивных функций $[8,9]$. Однако, как указывается в обзоре [10], отсутствие стандартов диагностики умеренных когнитивных нарушений не позволяет корректно сопоставлять результаты исследований когнитивной дисфункции при фибрилляции предсердий и определять зависимость степени снижения когнитивных функций от длительности нарушения сердечного ритма, т.е. постоянной или пароксизмальной форм фибрилляции предсердий.

При этом для оценки степени снижения когнитивной функции на помощь неврологу могут прийти нейрофизиологические маркеры когнитивных нарушений, проявляющиеся в ЭЭГ при ритмической фотостимуляции, и позволяющие оценить перестройку электрической активности мозга в соответствии с частотой, равной частоте световых импульсов. Такими маркерами могут быть, например, значения параметров фазовой синхронизации между паттернами ЭЭГ и ритмическими стимулами определенных частот. Так, в работе [11] на основании анализа синхронизации, выполненного с помощью синхросжатого вейвлет-преобразования с последующей оценкой мгновенных частот и фаз, мы продемонстрировали возможность выявления достоверных различий в параметрах фазовой синхронизации в двух группах лиц с хронически повышенным артериальным давлением, имеющих и не имеющих умеренные нарушения когнитивных функций. Было показано, что умеренные когнитивные нарушения при повышенном артериальном 
давлении коррелируют с большей длительностью фазовой синхронизации между ритмической фотостимуляцией и ответами мозга, а также со сдвигом этих ответов в более низкочастотный диапазон по сравнению с частотой возбуждения.

Целью настоящей работы является применение совместного рекуррентного анализа для оценки фазовой синхронизации между ритмической фотостимуляцией и ответами мозга у лиц с фибрилляцией предсердий и выявление различий в фазовой синхронизации при фибрилляции предсердий пароксизмального и постоянного типа при наличии проявлений умеренных когнитивных нарушений и без таких проявлений.

Для проверки устойчивости метода анализа совместных рекуррентностей к шуму в первой части работы была использована модель двух взаимодействующих хаотических осцилляторов без шума и после зашумления, а во второй части работы метод был применен к экспериментальным электрофизиологическим данным.

\section{1. Методы}

\section{1. Экспериментальные данные}

В качестве экспериментальных данных использованы записи ЭЭГ 35 пациентов (мужчин) с основным диагнозом „ишемическая болезнь сердца“. Данные были предоставлены клиникой военно-морской терапии Военно-медицинской академии, исследование было одобрено местным комитетом по этике. В анамнезе всех пациентов отсутствовали инсульты и инфаркты, болезни Альцгеймера и Паркинсона. Все пациенты имели нарушения сердечного ритма по типу мерцательной аритмии. При этом сердечная недостаточность не превышала первой стадии (по классификации сердечной недостаточности, предложенной Нью-Йоркской Ассоциацией кардиологов), т. е. без ограничений физической активности. Из них 19 человек имели пароксизмальную форму фибрилляции предсердий (группа 1, средний возраст $60.5 \pm 4.7$ лет) и 18 человек - постоянную форму фибрилляции предсердий (группа 2, средний возраст $65.5 \pm 6.7$ лет).

Для всех пациентов были характерны снижение памяти и быстрая утомляемость, по данным МРТ умеренные нарушения функционального состояния мозга, соответствующие начальной стадии сосудистой энцефалопатии (нарушения мозгового кровообращения). У 9 человек из группы 1 и 8 человек из группы 2 наблюдались начальные проявления умеренных когнитивных расстройств. В связи с этим все тестируемые пациенты были разделены на четыре группы: группа 1 и группа $1 d$ - пароксизмальная форма фибрилляции предсердий без и с когнитивными нарушениями соответственно, группа 2 и группа $2 d-$ постоянная форма фибрилляции предсердий без и с когнитивными нарушениями соответственно. Степень снижения когнитивных функций в группе $2 d$ была выше, чем в группе $1 d$ (в среднем 23.4 и 26.7 баллов по шкале MMSE (MiniMental State Examination), описывающей ориентацию во времени и пространстве, внимание, запоминание и понимание услышанного).

В контрольной группе (10 человек, средний возраст $33.2 \pm 5.6$ лет) нарушения сердечного ритма и когнитивные нарушения отсутствовали.

Записи содержали безартефактные фрагменты ЭЭГ, зарегистрированные в затылочных локусах O1, O2, Oz до, во время и после ритмической фотостимуляции, представляющей собой последовательность восьмисекундных серий световых импульсов с разной частотой с интервалом $30 \mathrm{~s}$ между сериями. Данные были подвергнуты цифровой фильтрации с использованием полосового фильтра $1-25 \mathrm{~Hz}$.

Фотостимул $p(t)$ аппроксимировался формулой

$$
p(t)=A\left(1+\sin \left(2 \pi f_{p} t\right)\right) .
$$

Так как нас будут интересовать только частотнофазовые характеристики отклика на фотостимул, постоянная составляющая в дальнейшем не рассматривается.

Для визуализации эволюции во времени ответа мозга на фотостимул были построены совместные рекуррентные диаграммы. Фазовая синхронизация между фотостимулом и ответом мозга на заданной частоте $f_{p}$ оценивалась методом совместного рекуррентного анализа на основании значения коэффициента взаимной корреляции между вероятностями рекуррентностей CPR.

\section{2. Построение совместных рекуррентных диаграмм и оценка фазовой синхронизации на основании анализа совместных рекуррентностей двух сигналов}

Совместная рекуррентная диаграмма является графическим представлением матрицы [12]:

$$
\begin{gathered}
J R_{i, j}(n, \varepsilon)=\Theta\left(\varepsilon-\left\|y_{i}-y_{j}\right\|\right) \Theta\left(\varepsilon-\left\|z_{i}-z_{j}\right\|\right), \\
i, j=1, \ldots, n
\end{gathered}
$$

где $y$ и $z$ - фазовые траектории, получаемые из исходных временных рядов $s(t)$ и $p(t), y_{i}$ и $y_{j}, z_{i}$ и $z_{j}$ - точки фазовых траекторий в моменты времени $t=i \delta t$ и $t=j \delta t$, где $\delta t-$ частота дискретизации временных рядов, $n$ - длина временных рядов $s(t)$ и $p(t)$, $m$ - оптимальная размерность вложения, символ \|\| обозначает норму, $\Theta-$ функция Хевисайда, $\varepsilon-$ радиус окрестности точек фазовой траектории.

Совместная рекуррентность отражает близость двух точек $z_{i}$ и $z_{j}$ фазовой траектории в моменты времени $t=i \delta t$ и $t=j \delta t$, т. е. нахождение этих точек в некоторой окрестности радиуса $\varepsilon$, и одновременно с этим близость двух точек $y_{i}$ и $y_{j}$ другой фазовой траектории в те же самые моменты времени $t$ [12]. 

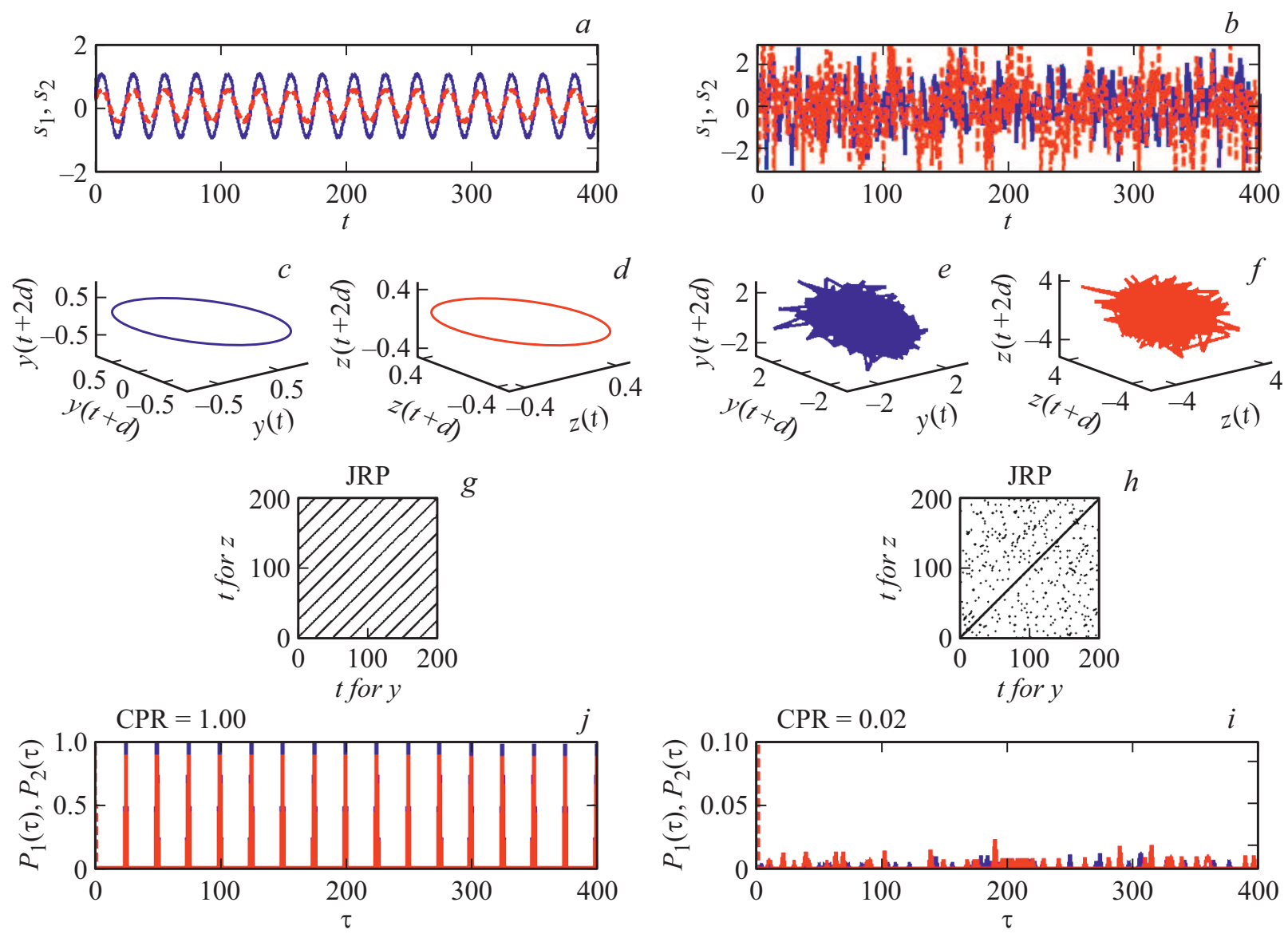

Рис. 1. Примеры периодических и стохастических сигналов $(a, b)$, их фазовых траекторий $(c-f)$, совместных рекуррентных диаграмм $(g, h)$ и гистограмм вероятностей рекуррентностей $(j, i)$.

Фазовые траектории получены из исходных временных рядов $s(t)$ и $p(t)$ с использованием метода временных задержек [13]. Этот метод позволяет отобразить анализируемые временные ряды $s(t)=\left\{s_{i}\right\}_{i=1}^{n}$ и $p(t)=\left\{p_{i}\right\}_{i=1}^{n}$, где $s_{i}=s\left(t_{i}\right), p_{i}=p\left(t_{i}\right), t_{i}=i \delta t$ в $m$-мерном пространстве таким образом, чтобы каждое значение этих рядов отображалось бы в точки этого пространства с координатами:

$$
\begin{gathered}
y\left(t_{i}\right)=\left(s\left(t_{i}\right), s\left(t_{i}+d\right), \ldots, s\left(t_{i}+(m-1) d\right),\right. \\
z\left(t_{i}\right)=\left(p\left(t_{i}\right), p\left(t_{i}+d\right), \ldots, p\left(t_{i}+(m-1) d\right),\right.
\end{gathered}
$$

где $d-$ величина задержки. Множества таких точек для последовательных моментов времени образуют траектории, описывающие эволюцию исследуемых временных рядов. При этом, согласно теореме Такенса, можно подобрать такую размерность пространства вложения $m$ и величину задержки $d$, чтобы полученное в результате преобразования множество точек сохраняло топологическую структуру исходного ряда [13]. Размерность пространства вложения представляет собой наименьшее число независимых переменных, однозначно определяющих его свойства.
Оптимальная временная задержка $d$ вычислялась путем нахождения минимума функции дивергенции распределений Кульбака-Лейблера [14]:

$$
S(d)=-\sum_{i, j} q_{i j}(d) \ln \left(\frac{q_{i j}(d)}{q_{i} q_{j}}\right)
$$

где $q_{i}-$ вероятность, с которой элемент временного ряда может оказаться в $i$-м временном интервале, $q_{j}$ в $j$-м интервале, $q_{i j}(d)-$ совместная вероятность того, что один элемент временного ряда окажется в $i$-м интервале, а другой, взятый с задержкой $d$, в $j$-м интервале.

Оптимальная размерность вложения $\mathrm{m}$ определялась методом минимизации числа ближайших ложных соседей [15]. Ложными ближайшими соседями считаются пары точек, для которых отношение между расстояниями $\frac{\left\|y_{i+1}-y_{j+1}\right\|}{\left\|y_{i}-y_{j}\right\|}$ до и после увеличения размерности вложения $m$ превышает некоторый порог. Поскольку число ложных соседей должно уменьшаться с увеличением значения $m$, построение функции зависимости числа ложных соседей от значения $m$ позволяет определить значение $m$, при котором число ложных соседей минимально. 

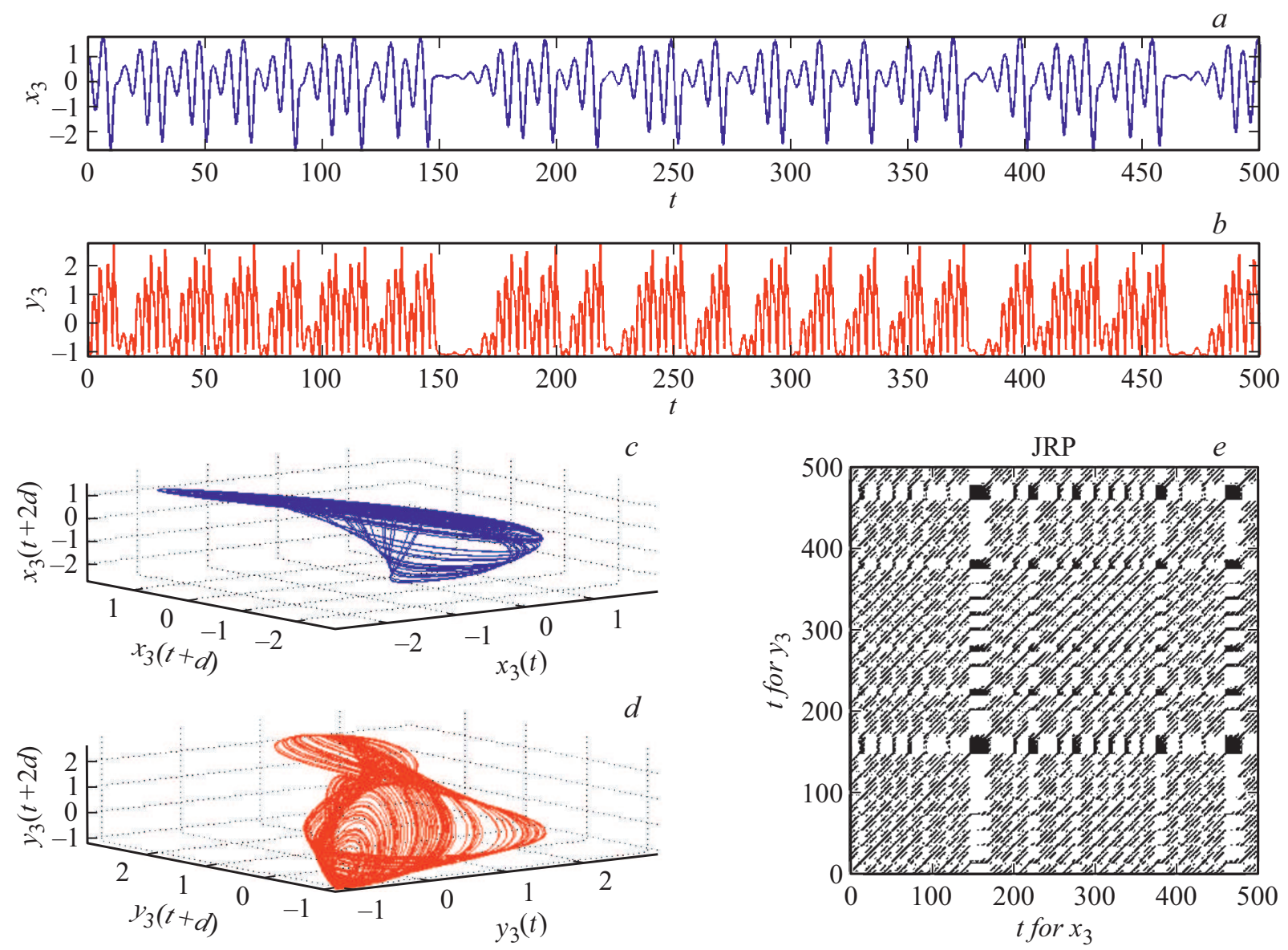

Рис. 2. Примеры колебаний синхронизированных осцилляторов $(a, b)$, их фазовых траекторий $(c, d)$ и совместная рекуррентная диаграмма $(e)$.

Наличие в совместной рекуррентной диаграмме диагональных линий, параллельных главной диагонали, показывает сходство динамического поведения двух колебательных процессов, при этом увеличение такого сходства вызывает увеличение числа рекуррентных точек вдоль главной диагонали, т.е. удлинение диагональных линий [12].

Для выявления временного совпадения динамики двух колебательных процессов в работе [5] предложено сравнение вероятностей рекуррентностей $P_{1}(\tau)$ и $P_{2}(\tau)$.

Вероятность рекуррентности $P(\tau)$ определяет возврат фазовой траектории $y(t)$ в окрестность предыдущего состояния после $\tau$ временных шагов и вычисляется как число точек, расположенных на диагональных линиях, отстоящих от главной диагонали на расстояние $\tau$ [5]:

$$
P(\tau)=\frac{1}{n-\tau} \sum_{i=1}^{n-\tau} R_{i, i+\tau},
$$

где

$$
R_{i, i+\tau}=\Theta\left(\varepsilon-\left\|y_{i}-y_{i+\tau}\right\|\right), \quad i=1, \ldots, n .
$$

В силу того что значение $R_{i, i+\tau}=1$ можно интерпретировать как приращение на $2 \pi$ фазы во временном интервале $\tau$, вероятность $P(\tau)$ рассматривается как статистическая мера частоты увеличения начальной фазы $\varphi$ на $2 \pi k$ в течение интервала времени $\tau$ [6]. Значение $k$ при этом соответствует количеству периодов, при которых выполняется условие

$$
\|y(\tau+T)-y(\tau)\|<\varepsilon,
$$

где $T$ - период колебаний фазовой траектории.

Поэтому совпадение положений максимумов функций $P_{1}(\tau)$ и $P_{2}(\tau)$ для двух фазовых траекторий $y$ и $z$ определяется как фазовая синхронизация двух временных рядов $s(t)$ и $p(t)$ [6]. В качестве меры фазовой синхронизации между двумя временными рядами в работе [5] был предложен коэффициент взаимной корреляции CPR (the correlation of probability of recurrence) между вероятностями рекуррентностей $P_{1}(\tau)$ и $P_{2}(\tau)$ :

$$
\mathrm{CPR}=\frac{1}{\sigma_{1} \sigma_{2}} \sum_{\tau=\tau_{e}}^{N / 2}\left(P_{1}(\tau)-\overline{P_{1}(\tau)}\right)\left(P_{2}(\tau)-\overline{P_{2}(\tau)}\right),
$$

где $\overline{P_{1}(\tau)}$ и $\overline{P_{2}(\tau)}-$ средние значения вероятностей, $\sigma_{1}$ и $\sigma_{2}-$ стандартные отклонения вероятностей $P_{1}(\tau)$ и $P_{2}(\tau), \tau_{e}$ - значение $\tau$, при котором $P_{i}(\tau)=1 / e$, $i=1,2$. 

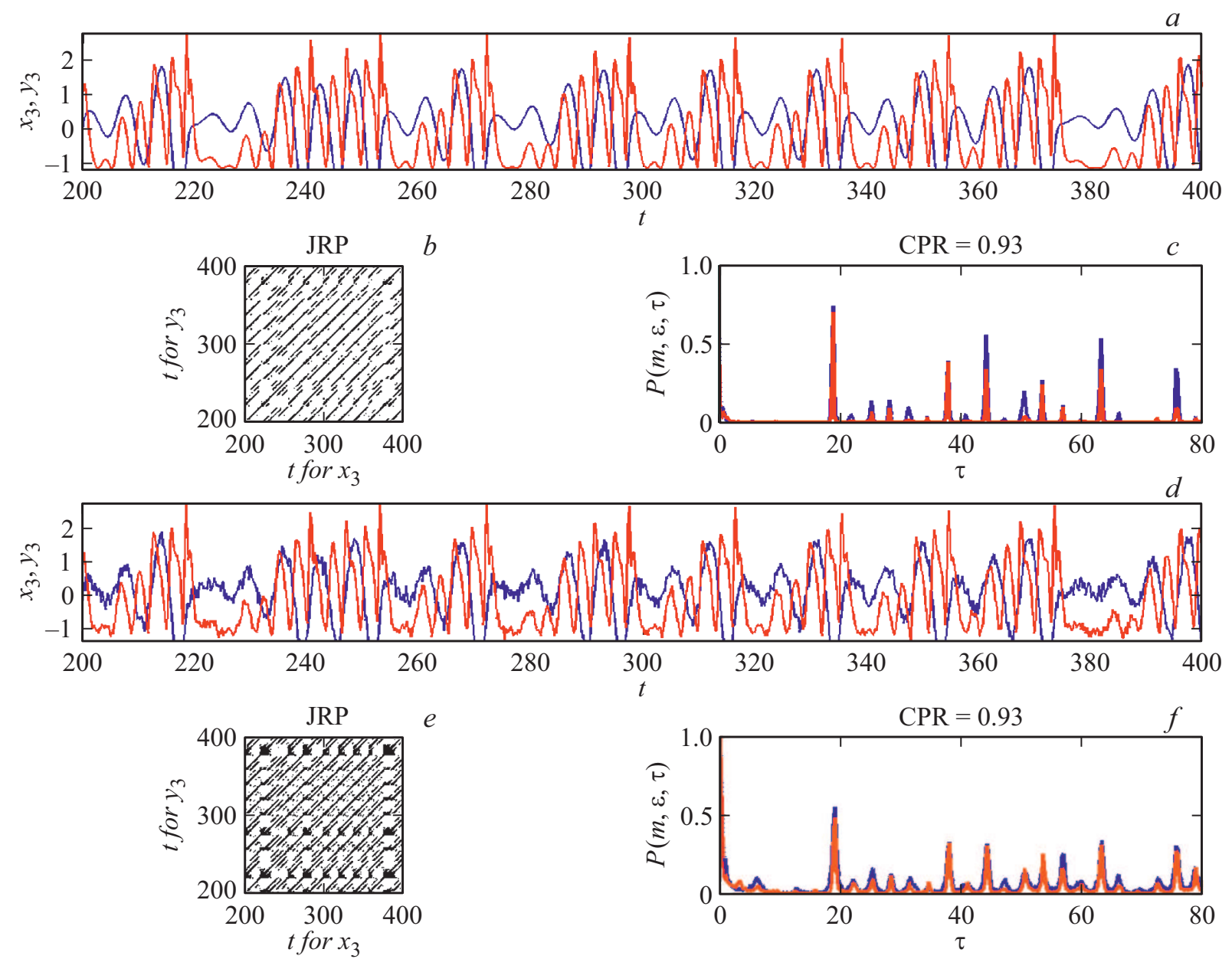

Рис. 3. Фрагменты колебаний синхронизированных осцилляторов без шума $(a)$ и после зашумления $(d)$, их рекуррентные диаграммы $(b, e)$ и вероятности рекуррентностей $(c, f)$.

Значение коэффициента взаимной корреляции CPR близко к единице, если два временных ряда находятся в идеальной фазовой синхронизации, поскольку в этом случае обе вероятности имеют максимумы в одинаковые моменты времени $k \tau$, где $k$ - целое число [5]. Если же два временных ряда несинхронизованы, максимумы вероятностей рекуррентностей возникают неодновременно. В этом случае значение CPR близко к нулю [5].

На рис. 1 представлены два периодических сигнала с одинаковой фазой и периодом, равным $25 \mathrm{~s}$ (рис. $1, a$ ), фазовые траектории для этих сигналов (рис. $1, c, d$ ) и рекуррентная диаграмма (рис. $1, g$ ), построенные при значениях $m=3$ и $d=8$, а также гистограмма вероятностей рекуррентностей (рис. $1, j$ ). Окрестность $\varepsilon$ задавалась с фиксированным числом ближайших точек $(\varepsilon=0.1)[5,12]$. Аналогичные построения выполнены на pис. $1, e, f, h, i$ (при $m=7$ и $d=12$ ) для двух стохастических сигналов. Для полностью синхронизованных сигналов рекуррентная диаграмма (рис. $1, g$ ) содержит длинные диагонали, находящиеся друг от друга на расстоянии, равном периоду колебаний, величина $\mathrm{CPR}=1$, (рис. $1, j)$. Для двух полностью стохастических сигналов (рис. $1, b)$ совместная рекуррентная диаграмма имеет только изолированные точки (рис. $1, h$ ), величина $\mathrm{CPR}=0.02$ (рис. $1, i)$. Таким образом, значение показателя CPR связано со сходством динамического поведения двух колебательных процессов (или отсутствием такого сходства).

Для выявления статистически значимых различий в средних значениях коэффициента взаимной корреляции CPR пяти рассмотренных групп использовался метод однофакторного дисперсионного анализа ANOVA с последующим множественным сравнением и проверкой парных гипотез по тесту Тьюки [16]. Если статистика, полученная по F-критерию Фишера, превышала критическое значение $F_{\text {crit }}=F_{4,35}=2.65$, то нулевая гипотеза о равенстве средних отвергалась. Значения 4 и 35 выбирались на основе того, что число групп $k=5$, число усредняемых значений в каждой группе 8 , общее число наблюдений $N=8 \cdot 5=40$, поэтому $k-1=4, N-k=35$. Статистически значимые отличия между группами определялись на основании значений $p<0.0051$ в силу того, что $n=k(k-1) / 2=10$ и $1-0.95^{1 / n}=0.0051$. 

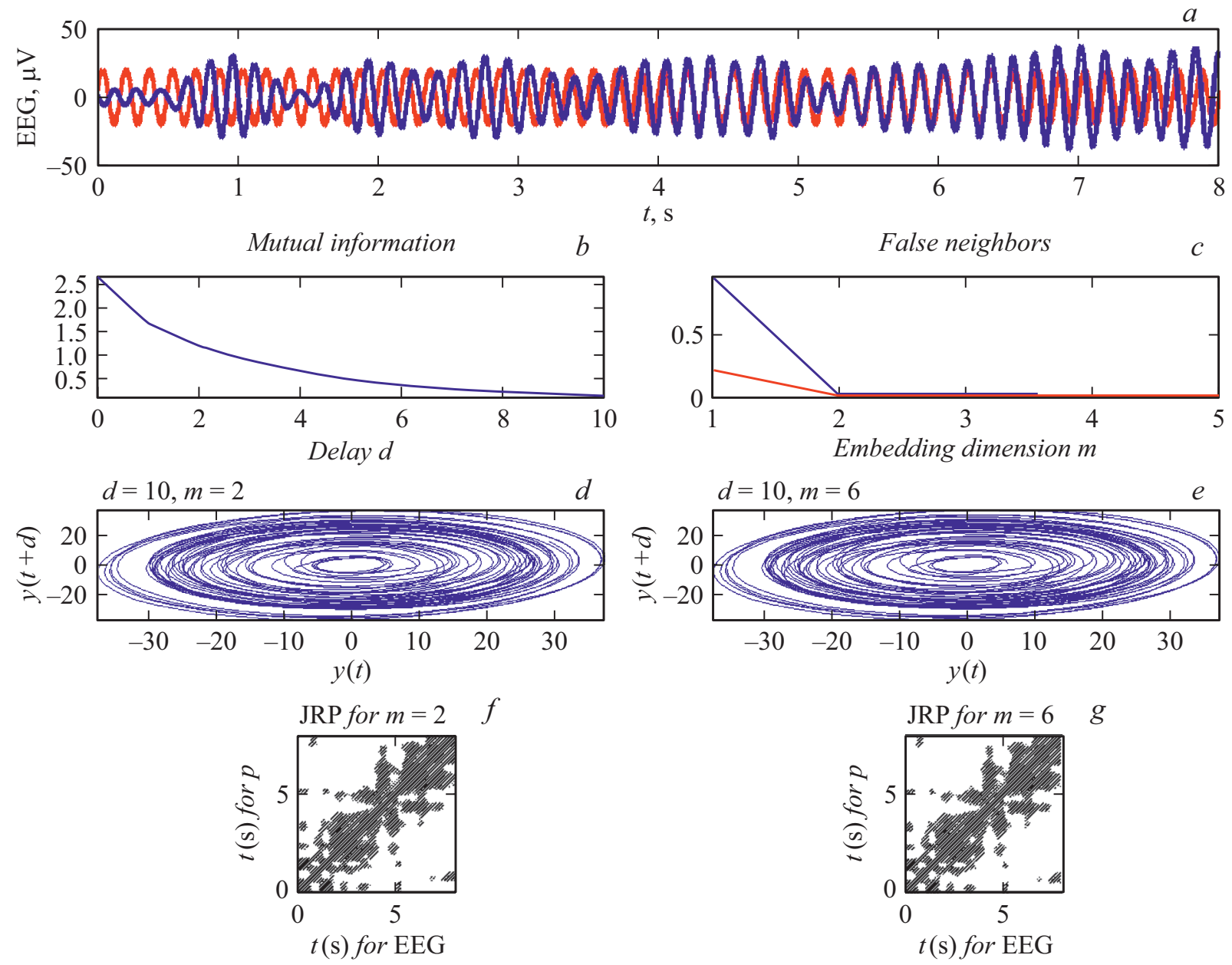

Рис. 4. Пример определения временной задержки $d(b)$ и размерности вложения $m(c)$ для фрагмента ЭЭГ (синяя кривая $(a)$ в online версии)) и построения фазовых траекторий этого фрагмента при $m=2(d)$ и $6(e)$ и совместных рекуррентных диаграмм для этого фрагмента и светового стимула $(f, g)$.

\section{2. Результаты}

\section{1. Анализ фазовой синхронизации колебаний двух взаимодействующих хаотических осцилляторов без и с шумовой составляющей}

Рассмотрим два взаимодействующих хаотических осциллятора, задаваемых системой уравнений Ресслера (9) и системой уравнений Лоренца (10):

$$
\begin{gathered}
\left\{\begin{array}{l}
d x_{1} / d t=b_{1}+x_{1}\left(x_{2}-c_{1}\right), \\
d x_{2} / d t=-x_{1}-x_{3}, \\
d x_{3} / d t=x_{2}+a_{1} x_{3},
\end{array}\right. \\
\left\{\begin{array}{l}
d y_{1} / d t=-a_{2}\left(y_{1}-y_{2}\right), \\
d y_{2} / d t=b_{2} u-y_{2}-u y_{3}, \\
d y_{3} / d t=u y_{2}-c_{2} y_{3}
\end{array}\right.
\end{gathered}
$$

Взаимодействие между этими системами осуществляется с помощью переменной $u=x_{1}+x_{2}+x_{3}$.
При значениях параметров $a_{1}=0.45, b_{1}=2, c_{1}=4$, $a_{2}=10, b_{2}=28, c_{2}=8 / 3$ эти системы находятся в состоянии обобщенной синхронизации [17]. Это отражается в совместных рекуррентностях двух осцилляторов [18].

На рис. 2, $a, b$ представлены компоненты $x_{3}(t)$ и $y_{3}(t)$, а на рис. 2, $c, d-$ реконструированные фазовые траектории, построенные по методу задержек при значениях параметра вложения $m=3$, времени задержки $d=5$ и $\varepsilon=0.1$. Несмотря на различия в форме реконструированных фазовых траекторий, две близкие точки фазовой траектории одной системы соответствуют двум близким точкам другой системы, что приводит к появлению совместных рекуррентностей в виде коротких диагональных линий на совместной рекуррентной диаграмме; увеличение сходства двух колебательных процессов вызывает увеличение числа рекуррентных точек вдоль главной диагонали, т.е. удлинение диагональных линий (рис. 2,e). Нерегулярное появление диагональных линий свидетельствует о хаотическом поведении осцилляторов. Скопление вертикальных и горизонтальных линий вбли- 

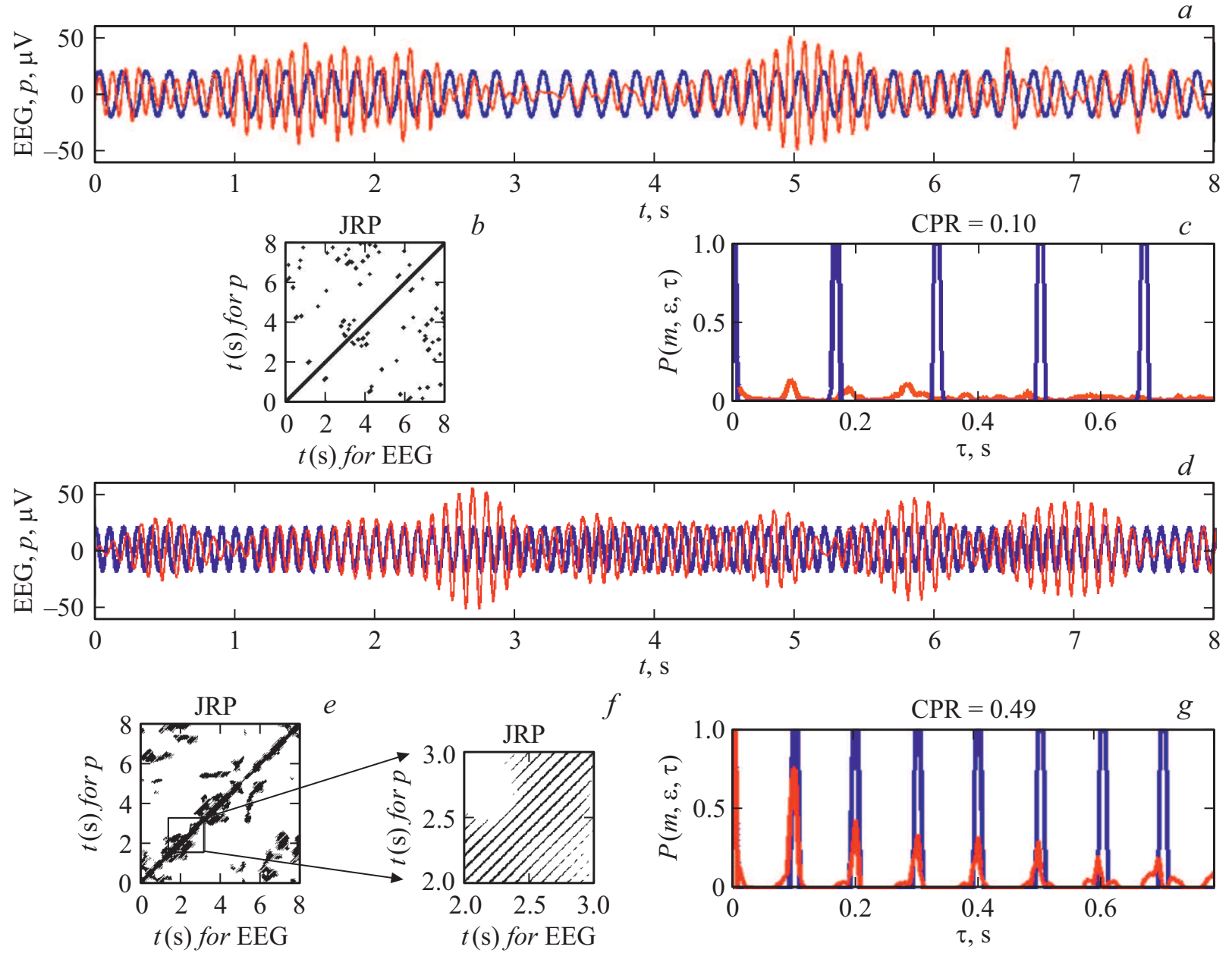

Рис. 5. Примеры отсутствия фазовой синхронизации между световым возбуждением на частоте 6 Нz и ответом мозга человека из контрольной группы $(a-c)$ и наличия синхронизации между возбуждением на частоте $10 \mathrm{~Hz}$ и ответом мозга этого человека $(d-g)$. $a, d$ - фрагменты ЭЭГ (красные кривые (в online версии)) и фотостимулы (голубые кривые (в online версии)); $b, e-$ совместные рекуррентные диаграммы; $f$ - увеличенный фрагмент рекуррентной диаграммы; $c, g$ - вероятности рекуррентностей для фотостимула (голубые кривые (в online версии)) и фрагментов ЭЭГ (красные кривые (в online версии)).

зи времен $t=150$ и 470 свидетельствует о том, что в эти интервалы времени динамическое состояние систем меняется незначительно, и они остаются ламинарными (замороженными).

На рис. 3, $a$ представлена часть траекторий $x_{3}(t)$ и $y_{3}(t)$ в интервале времени $t=[200,400]$, а на рис. $3, b-$ соответствующая этому временному интервалу совместная рекуррентная диаграмма, демонстрирующая наличие множества диагональных линий, свидетельствующих о временном совпадении динамики двух колебательных хаотических процессов через временные промежутки, равные 19, 38, 44, 63 и 75. Это подтверждается наличием в эти времена максимумов вероятностей рекуррентностей $P_{1}(\tau)$ и $P_{2}(\tau)$ (рис. $\left.3, c\right)$. Значение коэффициента взаимной корреляции, полученное на основе анализа рекуррентностей $(\mathrm{CPR}=0.93)$ свидетельствует о высокой степени фазовой синхронизации двух осцилляторов.
На рис. 3, $d$ изображены зашумленные на 25\% траектории $x_{3}(t)$ и $y_{3}(t)$, т. е. траектории $x_{3}(t)+0.25 \sigma_{3 x} \eta(t)$, $y_{3}(t)+0.25 \sigma_{3 y} \eta(t)$, где $\sigma_{3 x}$ и $\sigma_{3 y}$ - стандартные отклонения компонент $x_{3}(t), \eta(t)$ - реализация гауссовского шума. На рис. $3, e$ и $f$ представлены совместная рекуррентная диаграмма $(m=6, d=5$ и $\varepsilon=0.1)$ и вероятности рекуррентностей $P_{1}(\tau)$ и $P_{2}(\tau)$ соответственно. Несмотря на зашумление, максимумы вероятностей по-прежнему совпадают, и значение коэффициента взаимной корреляции не изменяется. Это свидетельствует об устойчивости метода анализа совместных рекуррентностей к шуму.

Устойчивость метода анализа совместных рекуррентностей к шуму описана также в работах $[5,6,18]$, где пронализировано влияние шума на определение величины CPR для множества хорошо известных взаимодействующих осцилляторов, таких как неидентичные системы Ресслера, системы Ресслера, находящиеся в фазово-когерентном режиме, система Лоренца под внешним 

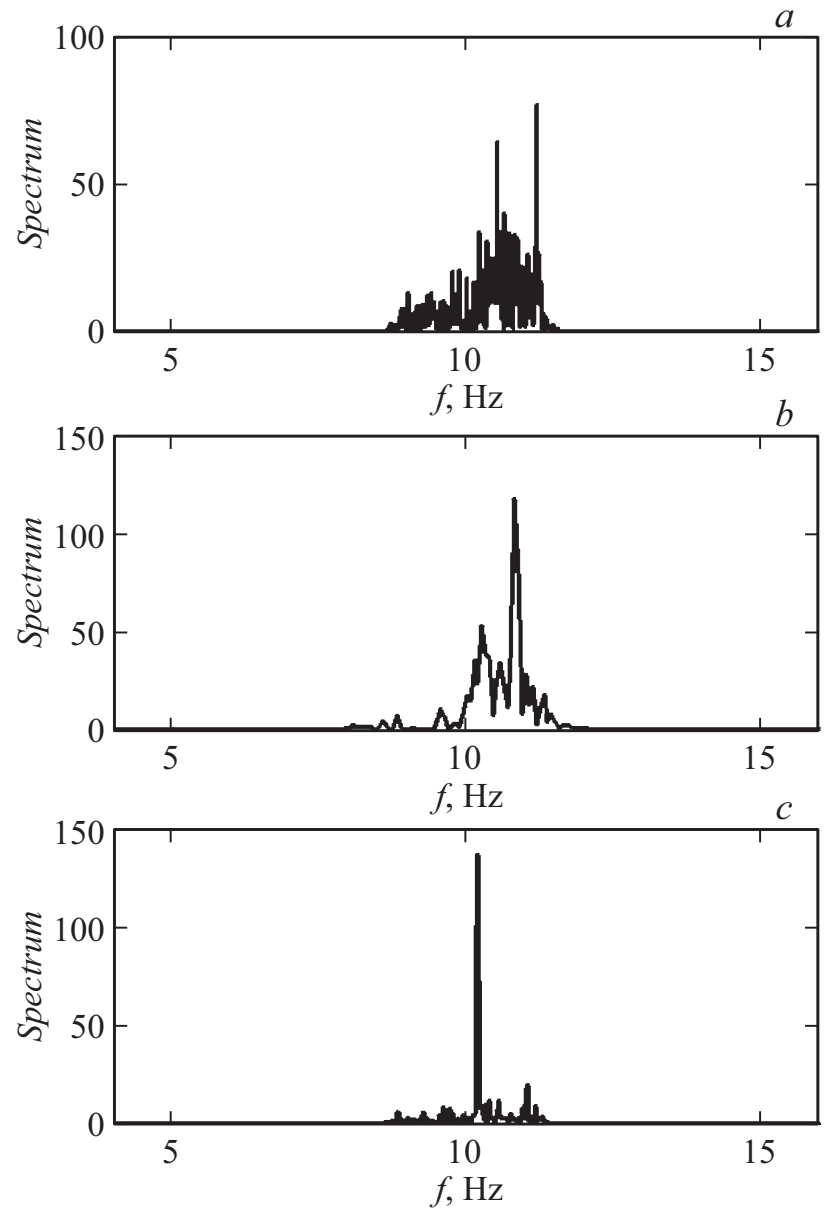

Pис. 6. Спектры ЭЭГ человека из контрольной группы до фотостимуляции $(a)$, во время стимуляции с частотой $6 \mathrm{~Hz}(b)$ и с частотой $10 \mathrm{~Hz}(c)$.

периодическим воздействием. В этих работах показано, что добавление к каждой анализируемой компоненте $80 \%$ гауссовского шума, т. е. переход, например, от $x_{3}(t)$ к $x_{3}(t)+0.8 \sigma_{3} \eta(t)$, где $\sigma_{3}-$ стандартное отклонение компоненты $x_{3}(t), \eta(t)$ - реализация гауссовского шума, не влияет на величину CPR, близкую к единице, при наличии фазовой синхронизации. Это объясняется тем, что эта величина связана не с локальными рекуррентностями в двух осцилляторах, а с усреднением рекуррентностей $[5,6,18]$.

\section{2. Анализ фазовой синхронизации фотостимулов и паттернов ЭЭГ}

В связи с отсутствием статистически значимых отличий в значениях коэффициента CPR для разных затылочных локусов ЭЭГ для дальнейшего анализа используются результаты, полученные для центрального затылочного локуса $\mathrm{Oz}$.

В связи с выделением паттернов ЭЭГ в узкой полосе частот вокруг частоты фотостимуляции $f_{p} \pm 1(\mathrm{~Hz})$, при построении совместных рекуррентных диаграмм величина оптимальной размерности вложения $m \leq 3$. Окрестность $\varepsilon$ выбиралась равной $1 \%$ от величины стандартного отклонения паттерна ЭЭГ.

Рис. 4 демонстрирует фазовые траектории, построенные для фрагмента ЭЭГ (синяя кривая на рис. 4, $a$ (в on line версии)) для значений $d=10, m=2$ (рис. $4, d$ ) и значений $d=10, m=6$ (рис. $4, e$ ), а также совместные рекуррентные диаграммы для этого фрагмента и светового стимула (красная кривая на рис. 4, $a$ (в on line версии)). Как видно из рис. 4, увеличение размерности пространства вложения в данном случае не влияет на совместную рекуррентную диаграмму. К тому же размерность $m=2$ совпадает для данного фрагмента ЭЭГ и фотостимула (нулевое число ложных соседей для синей и красной кривых на рис. 4, $c$ (в on line версии)).

На рис. 5, a, $d$ приведены фрагменты ЭЭГ, полученные для человека из контрольной группы во время ритмической стимуляции на частотах 6 и $10 \mathrm{~Hz}$ соответственно. Там же показаны соответствующие сигналы фотостимулов.

До фотостимуляции у этого человека в спектре фрагмента ЭЭГ преобладали частоты в интервале от 8 до $12 \mathrm{~Hz}$ (рис. 6,a), т. е. в альфа-диапазоне, характерном для состояния покоя; при стимуляции на частоте $6 \mathrm{~Hz}$ наблюдались частоты в интервале от 9 до $12 \mathrm{~Hz}$ (рис. $6, b)$, а при стимуляции на частоте $10 \mathrm{~Hz}$ - узкая полоса около $10 \mathrm{~Hz}$ (рис. $6, c)$. При стимуляции частотой $10 \mathrm{~Hz}$ наблюдается подстройка частоты в начале интервала стимуляции от 1.6 до $3 \mathrm{~s}$ и в конце - от 7 до $8 \mathrm{~s}$ (рис. 5,d). При возбуждении частотой $6 \mathrm{~Hz}$ такой подстройки не наблюдается (рис. 5,a).

Совместная рекуррентная диаграмма фотостимула с частотой $6 \mathrm{~Hz}$ и паттерна ЭЭГ (рис. 5,b) содержит в основном изолированные точки, т.е. не имеет совместных рекуррентностей для данного светового сигнала и фрагмента ЭЭГ. В отличие от этого, при частоте фотостимуляции $10 \mathrm{~Hz}$ совместная рекуррентная диаграмма содержит короткие линии (рис. 5,e). Для того чтобы детально показать эти прерывистые диагональные линии, на рекуррентной диаграмме (рис. 5,e) выделен короткий фрагмент длительностью $1 \mathrm{~s}$ (рис. 5,f). На этом фрагменте различаются диагональные линии, параллельные главной диагонали и расположенные друг от друга на расстоянии, совпадающем с размером лага $\tau=N_{\tau} \delta t=0.1 \mathrm{~s}$, видимом на построенной гистограмме вероятности рекуррентностей $P_{1}(\tau)$ для светового сигнала (синяя кривая на рис. $5, g$ (в on line версии)). Как видно на рис. $5, g$, вероятности $P_{1}(\tau)$ и $P_{2}(\tau)$ рекуррентностей светового сигнала и паттерна ЭЭГ (синяя и красная кривые соответственно (в on line версии)) имеют совпадающие максимумы, находящиеся на расстояниях, равных периодам колебаний светового сигнала $\left(\tau=T_{p}=1 / f_{p}=0.1 \mathrm{~s}\right)$. Значение коэффициента взаимной корреляции, полученное на основе анализа рекуррентностей, $\mathrm{CPR}=0.49$, свидетельствует о фазовой синхронизации между возбуждением на частоте $10 \mathrm{~Hz}$ и ответом мозга человека из контрольной 

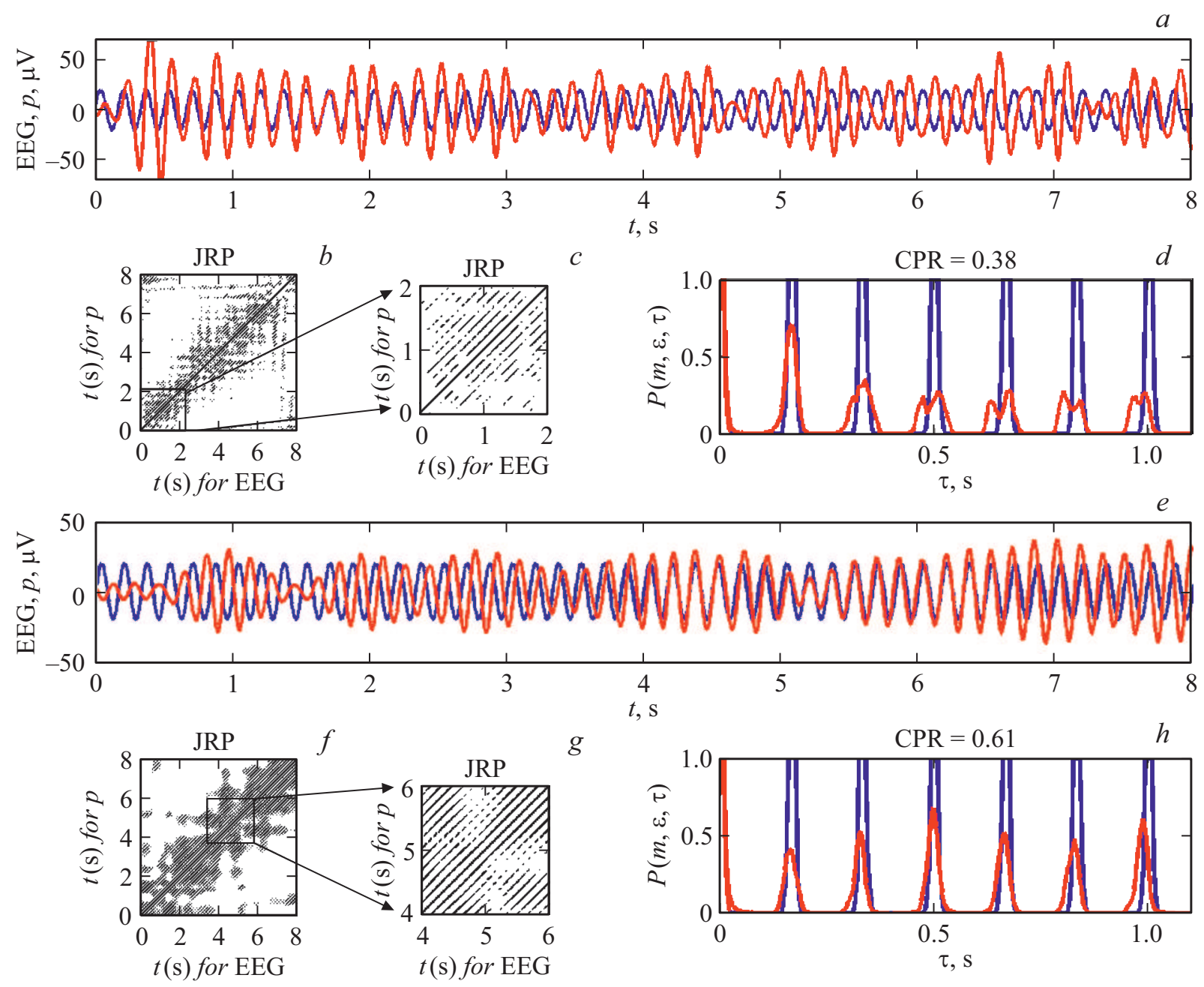

Рис. 7. Примеры фазовой синхронизации между световым возбуждением на частоте $6 \mathrm{~Hz}$ и ответом мозга человека из группы $1(a-d)$ и из группы $1 d(e-h)$. a,e - фрагменты ЭЭГ и фотостимулы; $b, f-$ совместные рекуррентные диаграммы; $c, g-$ их увеличенные фрагменты; $d, h-$ вероятности рекуррентностей для фотостимула и фрагментов ЭЭГ.

группы. Гистограмма вероятностей рекуррентностей для светового сигнала частоты $6 \mathrm{~Hz}$ и ответа мозга этого человека (рис. 5,c) показывает отсутствие одновременных совпадений максимумов вероятностей и значение коэффициента CPR, близкое к нулю, что свидетельствует об отсутствии фазовой синхронизации между возбуждением на этой частоте и ответом мозга.

Примеры разных степеней фазовой синхронизации между световым возбуждением на частоте $6 \mathrm{~Hz}$ и реакцией мозга человека с мерцательной аритмией пароксизмальной формы и отсутствием проявлений когнитивных нарушений (из группы 1) и человека с такой же формой аритмии, но с умеренными когнитивными нарушениями (из группы $1 d$ ) представлены на рис. 7. В первом случае синхронизация между сигналом и ответом наблюдается в первые $2.5 \mathrm{~s}$ после начала фотостимуляции (рис. 7,a), во втором - в интервале от 4 до $7 \mathrm{~s}$ (рис. 7,e). Это соответствует появлению эквидистантных диагональных линий на совместных рекуррентных диаграммах (рис. 7, b, $c, f, g$ ). Увеличенные фрагменты рекуррентных диаграмм демонстрируют увеличение числа и длины диагональных линий для второго случая. Рис. 7, $h$ показывает большее совпадение положения максимумов вероятностей рекуррентностей для светового стимула и реакции мозга при одних и тех же значениях лага $\tau=T_{p}=1 / f_{p}=0.16 \mathrm{~s}$ во втором случае по сравнению с первым. Значение коэффициента взаимной корреляции, $\mathrm{CPR}=0.61$, также выше во втором случае, чем в первом $(\mathrm{CPR}=0.38)$. Таким образом, в рассмотренном примере отклик мозга человека с мерцательной аритмией пароксизмальной формы на световое возбуждение на частоте $6 \mathrm{~Hz}$ при отсутствии когнитивных нарушений характеризуется меньшей степенью синхронизации между стимулом и ответом, чем в случае наличия умеренных когнитивных нарушений.

В отличие от этого для реакции мозга человека с мерцательной аритмией пароксизмальной формы на возбуждение на частоте $10 \mathrm{~Hz}$ наблюдается большая степень фазовой синхронизации в случае отсутствия умеренных когнитивных нарушений (рис. 8). Так, для 

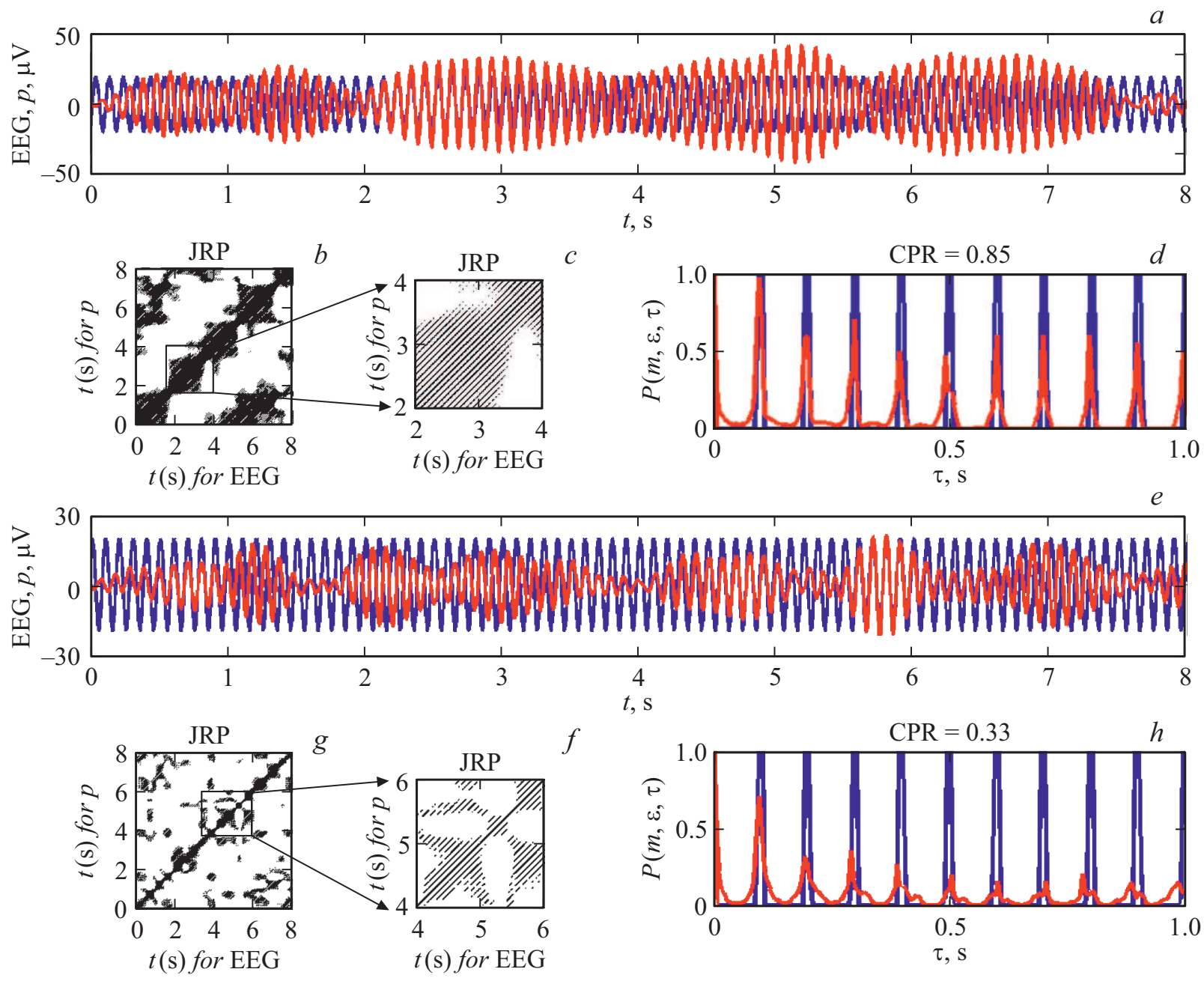

Рис. 8. Примеры фазовой синхронизации между световым возбуждением на частоте $10 \mathrm{~Hz}$ и ответом мозга человека из группы $1(a-d)$ и из группы $1 d(e-h)$. a,e - фрагменты ЭЭГ и фотостимулы; $b, f-$ совместные рекуррентные диаграммы; $c, g-$ их увеличенные фрагменты; $d, h-$ вероятности рекуррентностей для фотостимула и фрагментов ЭЭГ.

человека из группы 1 значение коэффициента взаимной корреляции $\mathrm{CPR}=0.85$, а для человека из группы $1 d$ это значение CPR $=0.33$ (рис. $8, d, h$ ).

На рис. 9 представлены данные о фазовой синхронизации между световым возбуждением на частоте $4 \mathrm{~Hz}$ и ответом мозга человека из группы 2 с мерцательной аритмией постоянной формы и отсутствием проявлений когнитивных нарушений (рис. 9, $a-d$ ) и из группы $2 d$ при наличии таких нарушений (рис. 9,e-h). Так же как и в случае аритмии пароксизмальной формы, умеренные когнитивные нарушения коррелируют с увеличением длины диагональных линий на рекуррентной диаграмме (рис. 9, $c, f)$ и увеличением значения коэффициента взаимной корреляции, $\mathrm{CPR}=0.95$ (рис. 9, $h$ ) вместо $\mathrm{CPR}=0.75$ (рис. 9, $d$ ).

Усредненные (по восьми пациентам в каждой группе) значения коэффициента взаимной корреляции CPR приведены в таблице. Для контрольной группы значение CPR близко к нулю для всех частот возбуждения, кроме альфа-диапазона $\left(\mathrm{CPR}=0.54 \pm 0.04, f_{p}=10 \mathrm{~Hz}\right.$ и $\mathrm{CPR}=0.6 \pm 0.04, f_{p}=12 \mathrm{~Hz}$ ). Это означает отсутствие фазовой синхронизации между реакцией мозга и фотостимулом всех частот, кроме альфа-диапазона.

Для группы 1 коэффициент взаимной корреляции имеет наибольшее значение для частоты $10 \mathrm{~Hz}$ $(\mathrm{CPR}=0.85 \pm 0.06)$, а для групп $1 d, 2$ и $2 d-$ для частот тета-диапазона $\left(\mathrm{CPR}=0.6 \pm 0.05, f_{p}=4 \mathrm{~Hz}\right.$, $\mathrm{CPR}=0.76 \pm 0.05, \quad f_{p}=6 \mathrm{~Hz} \quad$ и $\quad \mathrm{CPR}=0.89 \pm 0.06$, $f_{p}=4 \mathrm{~Hz}$ соответственно (см. таблицу)).

Однофакторный дисперсионный анализ выявил статистически значимые отличия в средних значениях коэффициента CPR в пяти рассмотренных группах для всех частот возбуждения. Статистика, полученная по F-критерию Фишера, превышает критическое значение $F_{\text {crit }}=F_{4,35}=2.65$ (см. таблицу). Уровень значимости критерия Фишера $(p)$, т.е. максимальная вероятность ошибочно отклонить нулевую гипотезу о равенстве средних, когда она верна, близок к нулю (см. таблицу).

Графические результаты однофакторного дисперсионного анализа для сравнения средних значений CPR в 

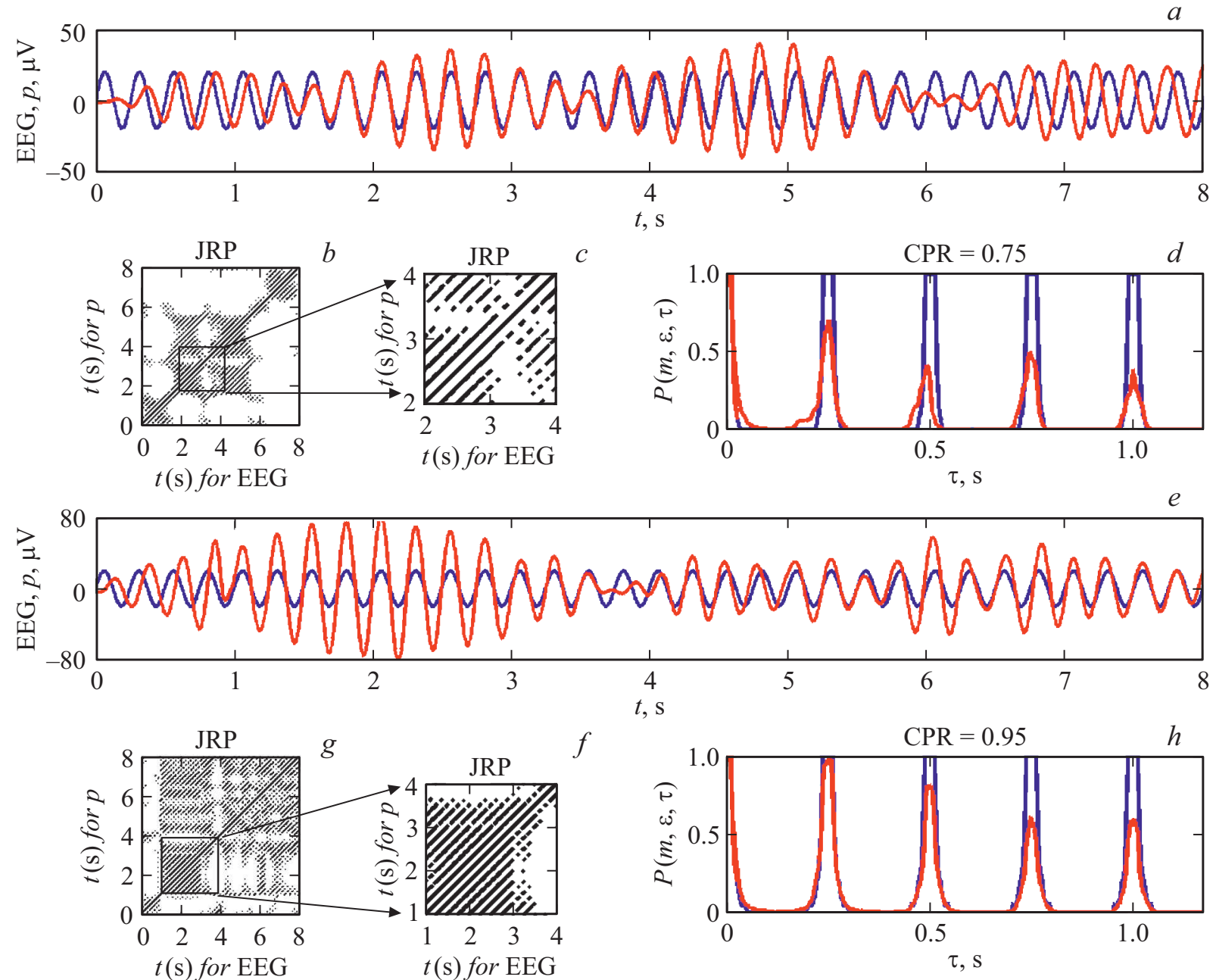

Рис. 9. Примеры фазовой синхронизации между световым возбуждением на частоте $4 \mathrm{~Hz}$ и ответом мозга человека из группы $2(a-d)$ и из группы $2 d(e-h)$. a,e - фрагменты ЭЭГ и фотостимулы; $b, f-$ совместные рекуррентные диаграммы; $c, g$ - их увеличенные фрагменты; $d, h-$ вероятности рекуррентностей для фотостимула и фрагментов ЭЭГ.

Средние значения коэффициента взаимной корреляции CPR для каждой группы, значения критерия Фишера $\left(F>F_{c r i t}\right.$, $\left.F_{c r i t}=F_{4,35}=2.65\right)$ и уровня значимости критерия $(p)$, вычисленные на основании однофакторного дисперсионного анализа

\begin{tabular}{r|c|c|c|c|c|c|c}
\hline $\begin{array}{c}f_{p}, \\
\mathrm{~Hz}\end{array}$ & $\begin{array}{c}\text { Контроль } \\
(N=8 / 10)\end{array}$ & $\begin{array}{c}\text { Группа 1 } \\
(N=8 / 10)\end{array}$ & $\begin{array}{c}\text { Группа } 1 d \\
(N=8 / 9)\end{array}$ & $\begin{array}{c}\text { Группа 2 } \\
(N=8 / 8)\end{array}$ & $\begin{array}{c}\text { Группа 2d } \\
(N=8 / 8)\end{array}$ & $F$ & $p$ \\
\hline 4 & $0.10 \pm 0.01$ & $0.39 \pm 0.04$ & $0.60 \pm 0.05$ & $0.74 \pm 0.03$ & $0.89 \pm 0.06$ & 285 & $2 \cdot 10^{-23}$ \\
6 & $0.12 \pm 0.01$ & $0.32 \pm 0.03$ & $0.58 \pm 0.05$ & $0.76 \pm 0.05$ & $0.87 \pm 0.6$ & 399 & $1.5 \cdot 10^{-25}$ \\
10 & $0.54 \pm 0.04$ & $0.85 \pm 0.06$ & $0.32 \pm 0.02$ & $0.73 \pm 0.05$ & $0.29 \pm 0.02$ & 309 & $1.7 \cdot 10^{-24}$ \\
12 & $0.60 \pm 0.04$ & $0.73 \pm 0.04$ & $0.22 \pm 0.02$ & $0.66 \pm 0.03$ & $0.37 \pm 0.03$ & 242 & $2 \cdot 10^{-22}$ \\
14 & $0.08 \pm 0.01$ & $0.63 \pm 0.02$ & $0.34 \pm 0.02$ & $0.19 \pm 0.02$ & $0.11 \pm 0.01$ & 409 & $4 \cdot 10^{-25}$ \\
16 & $0.09 \pm 0.01$ & $0.74 \pm 0.04$ & $0.15 \pm 0.02$ & $0.26 \pm 0.03$ & $0.10 \pm 0.01$ & 597 & $4 \cdot 10^{-28}$
\end{tabular}

пяти группах для различных частот возбуждения 4, 6, 12 и $16 \mathrm{~Hz}$ представлены на рис. 10. Большие различия в центральных линиях (медианах выборочных значений коэффициента (PR), соответствующие большим значениям статистики Фишера $F$, свидетельствуют о значительных различиях в групповых средних для всех рассмотренных частот возбуждения.
При этом длительность существования фибрилляции предсердий коррелирует с повышением значений CPR в ответах мозга на частоты тета-диапазона и с уменьшением в ответах на частоты в альфа- и бета-диапазонах. То есть при мерцательной аритмии постоянного типа получены большие, по сравнению с мерцательной аритмией пароксизмального типа, значения коэффициента 

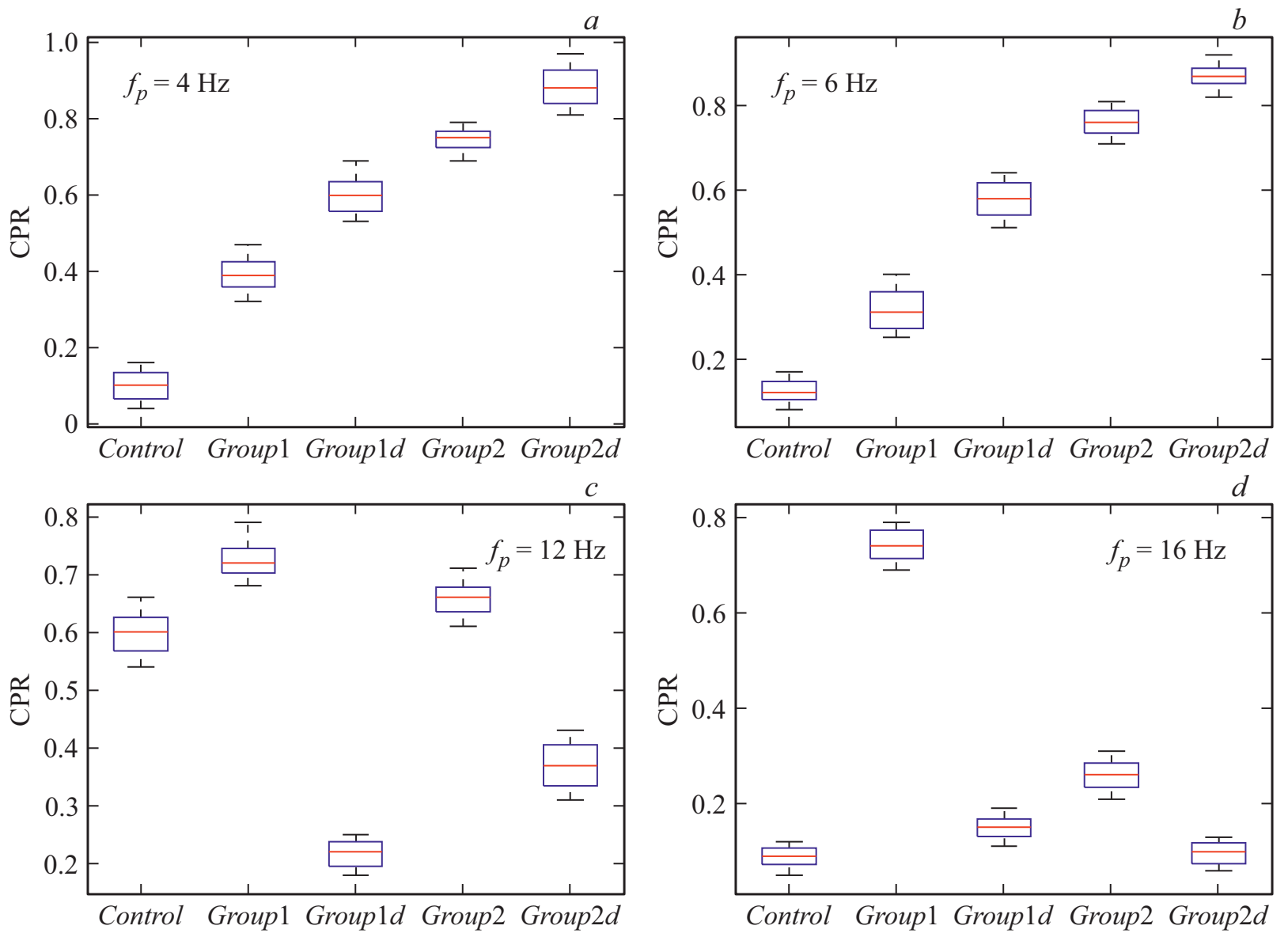

Рис. 10. Результаты однофакторного дисперсионного анализа для сравнения средних значений коэффициента взаимной корреляции CPR в пяти группах для различных частот возбуждения $4,6,12$ и $16 \mathrm{~Hz}(a-d)$.

CPR для частот тета-диапазона (рис. 10,a,b) и меньшие значения этого коэффициента для частот альфа- и бета-диапазонов (рис. $10, c, d)$.

Попарные сравнения средних значений коэффициента CPR для групп 1 и $1 d$, полученные на основании множественного сравнения с последующей проверкой парных гипотез по тесту Тьюки, показывают, что для всех частот возбуждения средние значения CPR для группы 1 и группы $1 d$ различаются на уровне значимости $p<0.0003$. При этом для частот 4 и $6 \mathrm{~Hz}$ значения CPR больше для группы $1 d$, а для частот 12 и $16 \mathrm{~Hz}$ меньше для этой группы, например, $\mathrm{CPR}=0.39 \pm 0.04$ и $\mathrm{CPR}=0.60 \pm 0.05, p=0.0002$ при $f_{p}=4 \mathrm{~Hz}$ и $\mathrm{CPR}=0.74 \pm 0.04$ и $\mathrm{CPR}=0.18 \pm 0.02$, $p=0.00012$ при $f_{p}=16 \mathrm{~Hz}$ для групп 1 и $1 d$ соответственно.

Аналогично этому большие значения CPR характерны для группы $2 d$, чем для группы 2 для частот 4 и $6 \mathrm{~Hz}$ и меньшие для частот 12 и $16 \mathrm{~Hz}$, например, $\mathrm{CPR}=0.74 \pm 0.03$ и $\mathrm{CPR}=0.89 \pm 0.06, p=0.0001$ при $f_{p}=4 \mathrm{~Hz}$ и $\mathrm{CPR}=0.26 \pm 0.03$ и $\mathrm{CPR}=0.1 \pm 0.02$, $p=0.00008$ при $f_{p}=16 \mathrm{~Hz}$ для групп 2 и $2 d$ соответственно.
Таким образом, при обеих формах мерцательной аритмии при умеренных когнитивных нарушениях наблюдается повышение значений коэффициента CPR для частот тета-диапазона и их уменьшение для частот альфа- и бета-диапазонов.

Поскольку степень снижения когнитивных функций в группе $2 d$ была выше, чем в группе $1 d$, полученные результаты показывают, что снижение когнитивных функций коррелирует с повышением степени синхронизации для частот возбуждения в тета- диапазоне и с ее понижением для частот альфа- и бета-диапазонов.

Ранее мы показали, что у пациентов с мерцательной аритмией, различающихся по длительности нарушения сердечного ритма, т.е. с постоянной или пароксизмальной формами фибрилляции предсердий, обнаруживаются достоверные различия в значениях коэффициентов усвоения и удержания ритма и временах его запоминания, вычисленных на основании вейвлетного анализа ритмических световых сигналов и паттернов ЭЭГ [19]. Также для фрагментов ЭЭГ при фибрилляции предсердий пароксизмального типа было характерно наличие совместных рекуррентностей в ответах мозга на частоты в тета- и альфа-диапазонах, а при фибрилляции 
предсердий постоянного типа - в основном только в тета-диапазоне. Однако в работе [19] при анализе рекуррентностей оценивалось только общее число рекуррентных точек в рекуррентной диаграмме. В отличие от этого в настоящей работе рассмотрены корреляции между вероятностями рекуррентностей для световых стимулов определенных частот и ответами мозга на них, т. е. рекуррентности в динамике и связанная с этой динамикой синхронизация сигналов.

Считается, что значительная реакция усвоения внешних ритмов различных частотных диапазонов связана с вовлечением большого количества нейронных ансамблей в патологический процесс, приводящий к разрушению внутренней синхронизации нейронных ансамблей и усилением их внешней синхронизации [20,21]. При этом наибольшее усиление внешней синхронизации в тета-диапазоне, характерное при снижении когнитивных функций у пациентов с постоянной формой мерцательной аритмии, может быть связано с хронической гипоксией мозга.

\section{Выводы}

Выполненное на основе совместного рекуррентного анализа исследование позволило выявить достоверные различия в степени фазовой синхронизации между ритмическими фотостимулами и электроэнцефалографическими паттернами у лиц с мерцательной аритмией пароксизмального и постоянного типа.

Показано, что постоянная форма мерцательной аритмии коррелирует с более высокими, по сравнению с пароксизмальной формой, значениями коэффициента взаимной корреляции между вероятностями рекуррентностей траекторий фотостимула для частот тета-диапазона и паттерна ЭЭГ и меньшими значениями этого коэффициента для частот альфа- и бета-диапазонов.

Умеренные когнитивные нарушения при обеих формах мерцательной аритмии коррелируют с повышением этого коэффициента (степени синхронизации) для частот тета-диапазона и понижением степени синхронизации для частот альфа- и бета-диапазонов. Степень снижения когнитивных функций также коррелирует с повышением степени синхронизации для частот тета-диапазона.

\section{Благодарности}

Авторы благодарят Н.Л. Гусеву за предоставленные экспериментальные записи ЭЭГ.

\section{Конфликт интересов}

Авторы заявляют, что у них нет конфликта интересов.

\section{Список литературы}

[1] E. Lowet, M.J. Roberts, P. Bonizzi, J. Karel, P. De Veerd. PLoS One, 11, 1 (2016). https://doi.org/10.1371/journal.pone.0146443

[2] B. Kralemann, M. Fruhwirth, A. Pikovsky, M. Rosenblum, Th. Kenner, J. Schaefer, M. Moser. Nature Commun., 4, 2418 (2013). DOI: $10.1038 /$ ncomms 3418

[3] A.E. Hramov, A.A. Koronovskii, V.I. Ponomarenko, M.D. Prokhorov. Phys. Rev. E, 73, 026208 (2006). DOI: 10.1103/PhysRevE.73.026208

[4] A.B. Bespyatov, M.B. Bodrov, V.I. Gridnev, V.I. Ponomarenko, M.D. Prokhorov. Nonlin. Phen. Compl. Syst., 6 (4), 885 (2003).

[5] M.C. Romano, M. Thiel, J. Kurths, I.Z. Kiss, J.L. Hudson. Europhys. Lett., 71 (3), 466 (2005).

[6] J. Kurths, M.C. Romano, M. Thiel, G.V. Osipov, M.V. Ivanchenko, I.Z. Kiss, J.L. Hudson. Nonlinear Dyn., 44, 135 (2006).

[7] D. Rangaprakash, N. Pradhan. Biomed. Signal Proces. Control, 11, 114 (2014).

[8] M. Acciarresi, M. Paciaroni, G. Agnelli, N. Falocci, V. Caso, C. Beccatini, S. Marcheselli, C. Rueckert, A. Pezzini, A. Morotti, P. Costa, A. Padovani, L. Csiba, L. Szabó, S.-Il Sohn, T. Tassinari, A.H. Abdul-Rahim, P. Michel, M. Cordier, P. Vanacker, S. Remillard, A. Alberti, M.P. Venti, C. D'Amore, U. Scoditti, L. Denti, G. Orlandi, A. Chiti, G. Gialdini, P. Bovi, M. Carletti, A. Rigatelli, J. Putaala, T. Tatlisumak, L. Masotti, G. Lorenzini, R. Tassi, F. Guideri, G. Martini, G. Tsivgoulis, K. Vadikolias, C. Liantinioti, F. Corea, M. Del Sette, W. Ageno, M.L. De Lodovici, G. Bono, A. Baldi, S. D'Anna, S. Sacco, A. Carolei, C. Tiseo, D. Imberti, D. Zabzuni, B.M. Doronin, V. Volodina, D. Consoli, F. Galati, A. Pieroni, D. Toni, S. Monaco, M.M. Baronello, K. Barlinn, L.-P. Pallesen, J. Barlinn, U. Bodechtel, J. Gerber, D. Deleu, G. Melikyan, F. Ibrahim, N. Akhtar, M.G. Mosconi, K.R. Lees. J. Stroke Cerebrovascular Diseases, 26, 1363 (2017).

[9] A. Singh-Manoux, A. Fayosse, S. Sabia, M. Canonoco, M. Bobak, A. Elbaz, M. Kivimäki, A. Dugravot. Eur. Heart J., 38, 2612 (2017).

[10] Е.3. Голухова, М.В. Шумилина, А.К. Кабисова. Креативная кардиология, 12, 31 (2018). DOI: 10.24022/1997-3187-2018-12-1-31-39. [E.Z. Goluchova, M.V. Shumilina, A.K. Kabisova. Creative Cardiology, 12, 31 (2018).]

[11] О.Е. Дик, А.Л. Глазов. ЖТФ, 91 (4), 678 (2021). DOI: $10.21883 /$ JTF.2021.04.50633.200-20

[12] N. Marwan, M.C. Romano, M. Thiel, J. Kurths. Phys. Reports, 438, 237 (2007). DOI: 10.1016/j.physrep.2006.11.001

[13] F. Takens. Dynamical Systems and Turbulence, Lecture Notes in Mathematics, D. Rand, L.S. Young, eds. (Springer-Verlag, Berlin, 1981)

[14] A.M. Fraser, H.L. Swinney. Phys. Rev., 33, 1134 (1986).

[15] M.B. Kennel, R. Brown, H.D. Abarbanel. Phys. Rev. A, 45, 3403 (1992).

[16] Y. Hochberg, A.C. Tamhane. Multiple Comparison Procedures (John Wiley \& Sons, Hoboken, N. J., 1987)

[17] L. Kocarev, U. Parlitz. Phys. Rev. Lett., 76, 1816 (1996). 
[18] M.C. Romano, M. Thiel, J. Kurths, C. Grebogi. Phys. Rev. E, 76, 036211-1 (2007)

[19] О.Е. Дик, И.А. Святогор, А.Д. Ноздрачев, Н.Л. Гусева. Физиология человека, $\mathbf{4 5}(1), 49$ (2019). DOI: 10.1134/S0131164619010053 [O.E. Dick, I.A. Svyatogor, A.D. Nozdrachev, N.L. Guseva. Human Physiology, 45, 40 (2019). DOI: $10.1134 / \mathrm{S} 0362119719010055]$

[20] A.I. Fedotchev, A.T. Bondar, S.G. Matrusov, V.S. Semenov, A.G. Soin. Usp. Physiol Nauk, 37, 82 (2006).

[21] O.E. Dick, I.A. Svyatogor. Neurocomputing, 165, 361 (2015). http://dx.doi.org/10.1016/j.neucom.2015.03.025 